\title{
Three Streptococcus pneumoniae sialidases; three different products
}

\author{
Guogang Xu, ${ }^{\dagger}$ Milton J. Kiefel, ${ }^{\ddagger}$ Jennifer C. Wilson, ${ }^{\ddagger}$ Peter W. Andrew, ${ }^{\natural}$ Marco R. Oggioni, ${ }^{\S}$ \\ and Garry L. Taylor ${ }^{\dagger}$ \\ ${ }^{\dagger}$ Biomolecular Sciences Research Complex, University of St Andrews, KY16 9ST, UK, ${ }^{\ddagger}$ Institute for Glycomics, Griffith \\ University Gold Coast Campus, Queensland 4222, Australia, "Department of Infection, Immunity and Inflammation, \\ University of Leicester, LE1 9HN, UK, ${ }^{\S}$ Dipartimento di Biologia Molecolare, Universita di Siena, 53100, Italy.
}

RECEIVED DATE (automatically inserted by publisher); glt2@st-andrews.ac.uk

\begin{abstract}
Streptococcus penumoniae is a major human pathogen responsible for respiratory tract infections, septicemia and meningitis and continues to produce numerous cases of disease with relatively high mortalities. S. pneumoniae encodes up to three sialidases; NanA, NanB and NanC, that have been implicated in pathogenesis and are potential drug targets. NanA has been shown to be a promiscuous sialidase, hydrolyzing the removal of Neu5Ac from a variety of glycoconjugates with retention of configuration at the anomeric centre, as we confirm by NMR. NanB is an intramolecular trans-sialidase producing 2,7-anhydro-Neu5Ac selectively from $\alpha 2,3$-sialosides. Here we show that the first product of NanC is 2-deoxy-2,3didehydro- $N$-acetylneuraminic acid (Neu5Ac2en) that can be slowly hydrated by the enzyme to Neu5Ac. We propose that the three pneumococcal sialidases share a common catalytic mechanism up to the final product formation step, and speculate on the roles of the enzymes in the lifecycle of the bacterium.
\end{abstract}

Sialic acids are a group of nine-carbon carboxylated sugars widespread on the cell surfaces of animal tissues ${ }^{1}$. The removal of sialic acids from various glycoconjugates by sialidases (neuraminidases) is a key biochemical event associated with infection, apoptosis and signaling ${ }^{2}$. As shown in Scheme 1, sialidases typically hydrolyze sialoglycosides to release $\mathrm{N}$-acetylneuraminic acid (Neu5Ac, 1). Trypanosomal trans-sialidases can also hydrolyze sialosides, but their major function is to transfer Neu5Ac from $\alpha 2,3$-sialosides to another sugar $^{3}$, while the leech intra-molecular (IT) trans-sialidase produces 2,7-anhydro-Neu5Ac (2), instead of Neu5Ac (1) ${ }^{4}$.

Streptococcus pneumoniae is a human pathogen responsible for respiratory tract infections, septicemia, and meningitis, and it expresses up to three sialidases, two of which (NanA, NanB) are validated pneumococcal virulence factors and potential drug targets ${ }^{5-7}$. All three sialidases possess a carbohydrate-binding module (CBM) in addition to the catalytic domain. A study of clinical isolates of $S$. pneumoniae showed NanA, NanB and NanC to be present in 100\%, 96\% and $51 \%$ of strains, respectively, with NanC more prevelant in cerebrospinal fluid ${ }^{7}$. NanA is anchored to the bacterium's outer membrane and its CBM has recently been shown to be a key determinant in enabling the bacterium to cross the bloodbrain barrier ${ }^{8}$. Despite the fact that these three pneumococcal sialidases are known virulence factors, the precise function and role of these enzymes is less well understood. Therefore, the aim of this study was to characterize the sialidase activities of the three sialidases by NMR and kinetics, to see if any variation may help explain different roles in pneumococcal infection.

NanA had previously been shown to cleave $\alpha 2,3-, \alpha 2,6-$ and $\alpha 2,8-$ linked sialic acids using the thiobarbituric (TBA) assay ${ }^{9}$. The ${ }^{1} \mathrm{H}$ NMR experiments herein show that NanA acts as a typical exo-sialidase, hydrolyzing $\alpha 2,3-$ or $\alpha 2,6-$ sialyllactose (3SL or 6SL) to release $\alpha$-Neu5Ac (1). In solution, 1 then gradually mutarotates to $\beta$-Neu5Ac (3), the more stable isoform (Figure 1a for 3SL, Figure S4.1 for 6SL). Our previous NMR studies showed NanB to be an intramolecular trans-sialidase producing 2,7-anhydro-Neu5Ac (2) from $\alpha 2,3$-sialosides ${ }^{9}$ and this has been confirmed in a

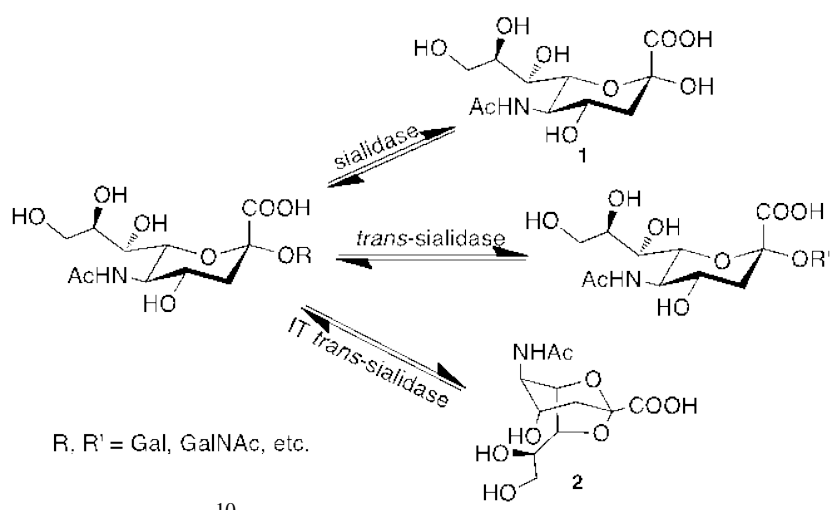

crystal structure $^{10}$.

Scheme 1. Reactions catalyzed by different sialidases.

The initial product of the NanC catalysed cleavage of 3SL surprisingly failed to react in the TBA assay. ${ }^{1} \mathrm{H}$ NMR experiments showed that NanC initially makes only 2-deoxy2,3-didehydro- $N$-acetylneuraminic acid (Neu5Ac2en, 4) from 3SL (but not from 6SL) (Figure 1b), that is unable to react with TBA reagents. Although the sialidases from influenza B virus and Vibrio cholerae have been shown to release very small amounts of Neu5Ac2en as a by-product ${ }^{11,12}$, NanC converts 3SL to Neu5Ac2en directly with a $k_{\text {cat }}$ of $\sim 19 \mathrm{~min}^{-1}$ (Table 1).

Neu5Ac2en is a general sialidase inhibitor thought to mimic the transition-state sialyl cation in the sialidase mechanism. It inhibits NanA and NanB with a $K_{i}$ of $2 \mu \mathrm{M}$ and $0.3 \mathrm{mM}$, respectively ${ }^{9}$. Upon 3SL depletion in the reaction, NanC starts to hydrate the accumulated Neu5Ac2en to Neu5Ac by 
attacking $\mathrm{C} 2$ at the axial position. In the ${ }^{1} \mathrm{H}$ NMR spectra (Figure 1b), this is reflected by the appearance after several hours of a unique doublet at $\delta 2.05$ ppm with a coupling constant of $5.05 \mathrm{~Hz}$. Note that H3eq appears as a doublet because the NanC catalysed hydration in $\mathrm{D}_{2} \mathrm{O}$ introduces an axial deuterium at C-3. This hydration reaction promoted by NanC was further confirmed by using pure Neu5Ac2en as the substrate (Figure S4.2). The NanC reaction with 3SL indicates this sialidase may adopt a ping-pong mechanism. Hydration of the alkene double bond by NanC is highly efficient and approximately 500-fold higher than that observed for Clostridium perfringens sialidase NanI. ${ }^{13}$ It is possible that the dual function of NanC, producing or eliminating Neu5Ac2en, may be dependent upon which substrate (a sialoside or Neu5Ac2en) is present in higher concentration.

(a)

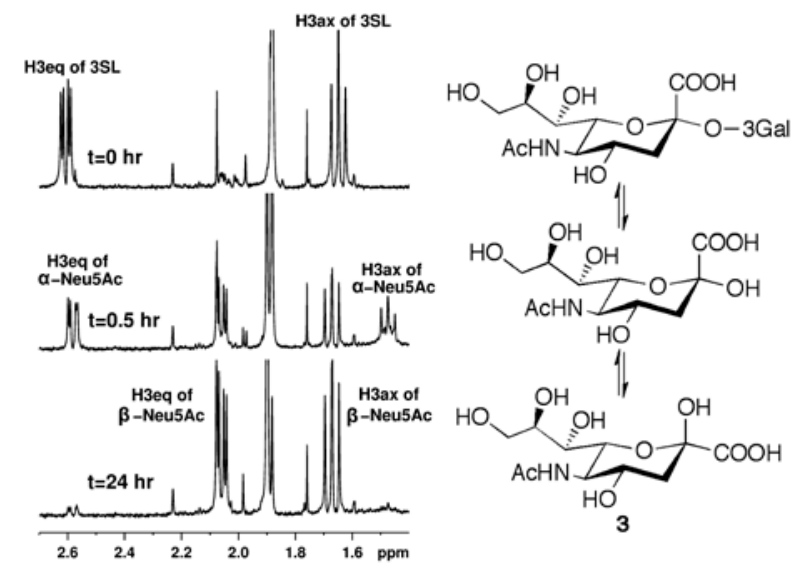

(b)

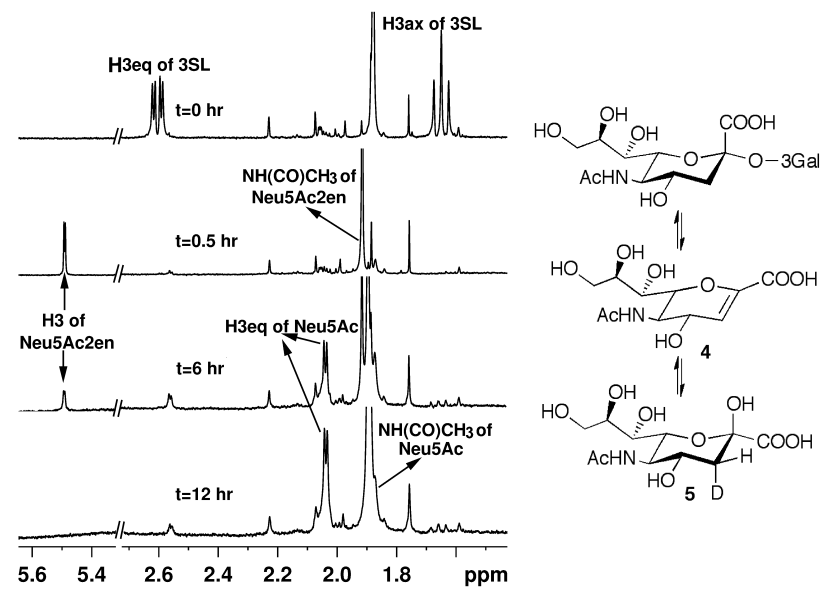

Figure 1. ${ }^{1} \mathrm{H}$ NMR spectroscopy studies of (a) NanA; and (b) NanC with $\alpha 2,3$-sialyllactose (3SL).

\begin{tabular}{|l|l|l|l|l|}
\hline & \multicolumn{2}{|c|}{$3 \mathrm{SL}$} & \multicolumn{2}{c|}{$6 \mathrm{SL}$} \\
\hline & $k_{\mathrm{cat}}\left(\mathrm{min}^{-1}\right)$ & $K_{\mathrm{m}}(\mathrm{mM})$ & $k_{\mathrm{cat}}\left(\mathrm{min}^{-1}\right)$ & $K_{\mathrm{m}}(\mathrm{mM})$ \\
\hline NanA & $892.8 \pm 15$. & $0.64 \pm 0.0$ & $916.5 \pm 27.3$ & $2.17 \pm 0.14$ \\
& 9 & 2 & & \\
\hline NanB & $56.5 \pm 6.4$ & $\begin{array}{l}2.39 \pm 0.1 \\
9\end{array}$ & $0.13 \pm 0.01$ & $0.15 \pm 0.04$ \\
& & $\begin{array}{l}1.08 \pm 0.3 \\
5\end{array}$ & \multicolumn{2}{c|}{ No activity } \\
\hline NanC & $18.8 \pm 3.1$ & \multicolumn{2}{|c|}{} \\
& & 5
\end{tabular}

Table 1. Kinetic analysis of the pneumococcal sialidases

Our ${ }^{1} \mathrm{H}$ NMR findings clearly show disparate catalytic properties of the three $S$. pneumoniae sialidases, which might play different but cooperative roles in pneumococcal virulence. NanA's role could be the first-line virulence factor for sialic acid removal; NanB produces 2,7-anhydro-Neu5Ac, which could be a nutritional source for the colonized bacteria; whereas NanC could be a regulator for NanA actions by producing or removing Neu5Ac2en from environments at different stages of pneumococcal infection.

We have previously reported the crystal structures of NanB and the catalytic domain of $\mathrm{NanA}^{9,14}$. A model structure of NanC was made based on $\mathrm{NanB}$, with which it shares $50 \%$ sequence identity. Structural comparisons show that NanA, NanB and NanC present significantly different topologies around the active site (Figure 2a). In NanA, the catalytic cavity is flat and open, allowing easy access for various substrates or water molecules to aid hydrolysis. However, both NanB and NanC present a relatively narrow cleft for catalysis, due to the presence of a bulky tryptophan (Trp674 and Trp716 in NanB and NanC, respectively). This tryptophan is conserved in the leech sialidase ${ }^{4}$ and $T$. cruzi trans-sialidase, ${ }^{3}$ where it provides a platform for an $\alpha 2,3$-linked galactose, and produces steric hindrance of $\alpha 2,6-$ and $\alpha 2,8$-linked substrates. Moreover, Phe396 and Trp581 in NanC would further contribute to a hydrophobic environment for catalysis. Figure $2 b$ depicts the superimposition of pneumococcal sialidases structures, and shows that the residues forming the hydrophobic pocket accommodating the acetamido group also vary significantly between the sialidases. 

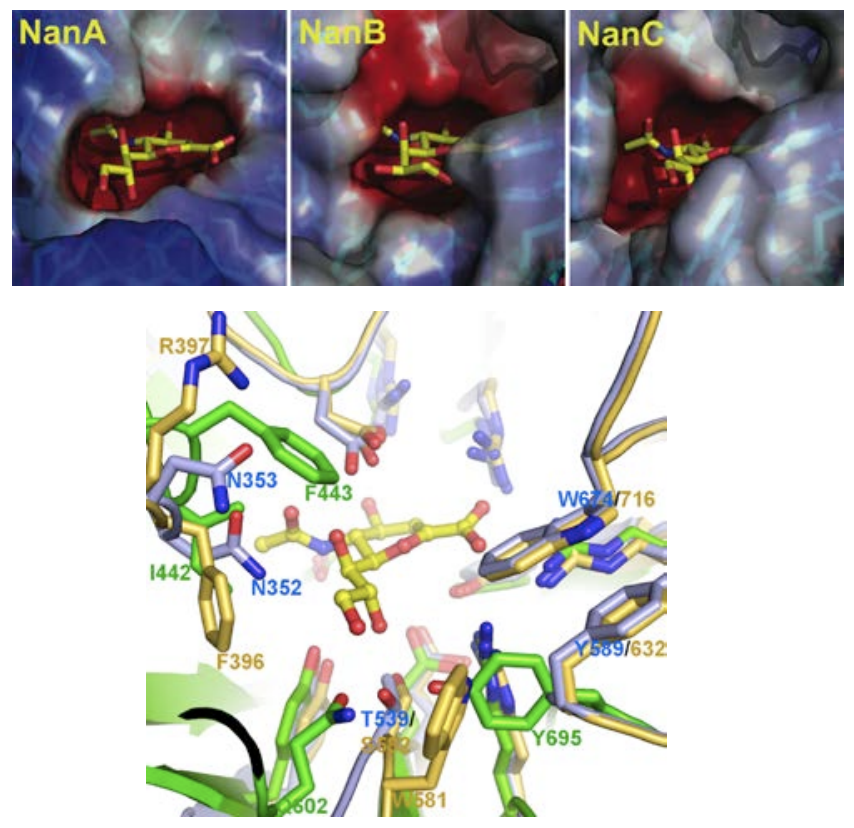

Figure 2 Active sites of pneumococcal sialidases in the presence of Neu5Ac2en. (a, top) Topology of active sites colored by electrostatic potential. (b, bottom) Structural comparison of NanA (green; PDB code: 2vvz), NanB (blue; 2vw0) and NanC model (gold).

In spite of these differences in hydrophobic residues within the catalytic domain, the three pneumococcal sialidases share the conserved catalytic residues including a tri-arginine cluster that interacts with the carboxylate group of sialic acids, a nucleophilic tyrosine with its associated glutamic acid residue, an aspartic acid above the sugar ring that acts as an acid/base, as well as a hydrophobic pocket accommodating the C-5 acetamido group (Figure 2b, Figure S3, Table S1). These are the features required for catalysis, which comprises a distortion of the sugar ring to a boat conformation $\left(\mathrm{B}_{2,5}\right)$ upon binding and subsequent formation of a transition state with the formation of the ${ }^{4} \mathrm{H}_{5}$ half-chair oxocarbonium ion or covalently-bonded intermediate in a ${ }^{2} \mathrm{C}_{5}$ chair form (Figure 3).

Given that the three pneumococcal sialidases have the requisite conserved catalytic residues to cleave sialosides, it is reasonable to conclude that it is the final product formation step which is different for each of the sialidases. As depicted in Figure 3, attack at C-2 position of the oxocarbonium ion by different nucleophiles, such as a water molecule in hydrolytic sialidase, or the O-7 atom of the sialyl cation in IT transsialidase or another carbohydrate acceptor such as $\beta \mathrm{Gal}$ in trans-sialidase, the final products could be Neu5Ac, Neu2,7anhydro5Ac or Neu5Ac- $\beta$ Gal. Here, S. pneumoniae NanA and NanB follow the mechanisms of a hydrolytic sialidase and an IT trans-sialidase, respectively.

Figure 3. The proposed mechanisms of NanA, NanB and NanC.

In the first reaction to produce Neu5Ac2en, NanC might also adopt the generalized mechanism involving the formation

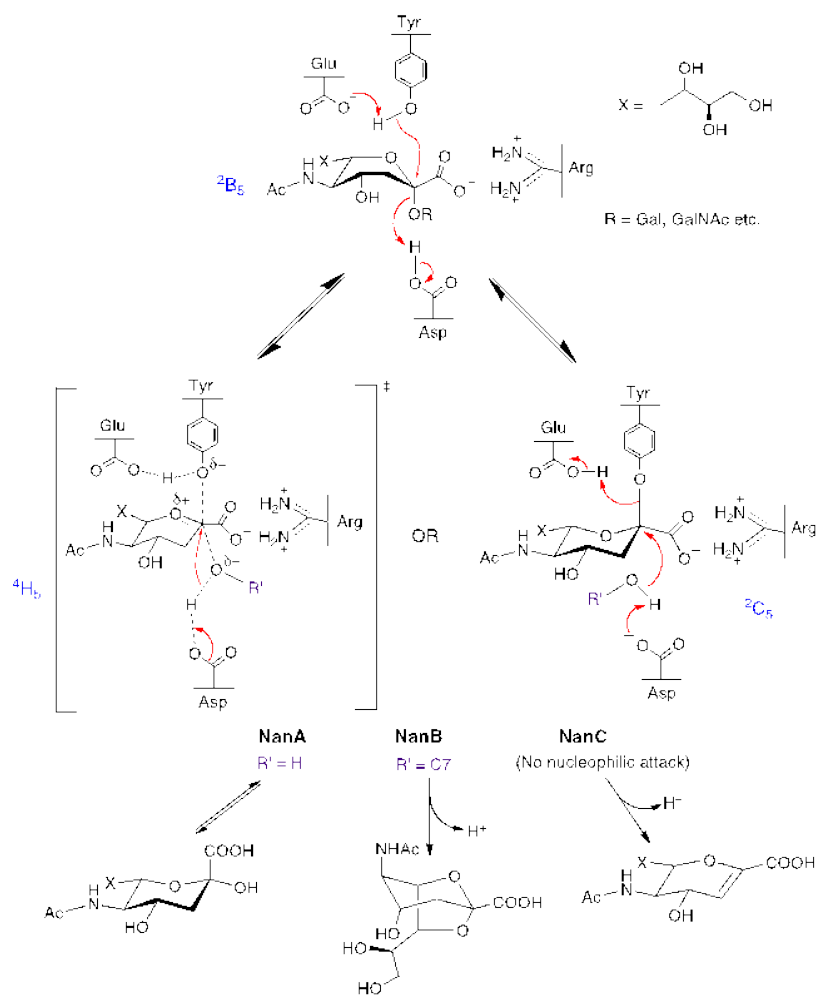

of a transition-state sialyl cation or a covalently sialylated enzyme intermediate (Figure 3). However, in contrast to NanA and NanB, there must be no effective nucleophilic attack happening in the active site of NanC. This may in part be attributable to the hydrophobic environment created by residues stacking around the catalytic cleft, as well as the leaving lactose group covering on top. Furthermore, NanC can only form limited hydrogen bonds with Neu5Ac2en with a low $k_{i}$ of $\sim 3 \mathrm{mM}$. This also makes the sialyl cation transition state less easily held by NanC, and it is therefore released as the transition-state analogue Neu5Ac2en (Figure 3). Mutations of Phe396 alone or with Trp581 allow NanC to produce some Neu5Ac, as well as Neu5Ac2en (Figures S4.3, S4.4).

The subsequent Neu5Ac2en hydration reaction probably only occurs at high concentrations of Neu5Ac2en, and presumably proceeds via a similar mechanism to that previously reported for catalysis by $C$. perfringens NanI. ${ }^{13}$ Briefly, the electrons in the nucleophilic $\pi$ bond could attack the proton of the catalytic aspartic acid (Asp315), resulting in the formation of an oxocarbonium ion intermediate. Without any lactose covering on top, water molecules could be readily picked up and activated by Asp315. The water attacks the positive charge at $\mathrm{C}-2$ position to produce a protonated intermediate, which transfers a proton back to Asp315, thus releasing Neu5Ac. An Asp315Ala mutant retains only 2\% activity and lacks Neu5Ac2en hydrating capacity (Figures S4.5, S4.6), further confirming the role of Asp315 in catalysis. To discount the possibility of an elimination reaction, a thiosialoside substrate was examined with NanC by NMR, but this showed no activity (Figure S4.7).

The findings reported here, as summarized in Figure 3, add further evidence to the currently proposed sialidase mechanisms. By adopting distinct catalytic mechanisms, NanA, NanB and NanC might work together to coordinate the sialidase action associated with pneumococcal virulence. Since each of pneumococcal sialidases are potential drug targets, our findings show that, for each of the three distinct 
sialidases, different strategies may need to be considered for ongoing structure-based drug design.

Acknowledgement. G.X. was supported by BioCryst Pharmaceuticals Inc. (Birmingham, AL). PWA and MO were funded by the EU (Pneumopep). Authors thank Dr. Uli Schwarz-Linek, Dr. Robert Hagan and Prof. Jim Naismith for kind help and useful discussions.

Supporting Information Available: Experimental details, ${ }^{1} \mathrm{H}$ NMR spectra and sequence alignment data. This material is available free of charge via the Internet at http://pubs.acs.org.

\section{References:}

(1) Kiefel, M. J.; von Itzstein, M. Chem Rev 2002, 102, 471-90.

(2) Taylor, G. Curr Opin Struct Biol 1996, 6, 830-7.

(3) Amaya, M. F.; Watts, A. G.; Damager, I.; Wehenkel, A.; Nguyen, T.; Buschiazzo, A.; Paris, G.; Frasch, A. C.; Withers, S. G.; Alzari, P. M. Structure 2004, 12, 775-84.

(4) Luo, Y.; Li, S. C.; Li, Y. T.; Luo, M. J Mol Biol 1999, 285, 323-32.

(5) Manco, S.; Hernon, F.; Yesilkaya, H.; Paton, J. C.; Andrew, P. W.; Kadioglu, A. Infect Immun 2006, 74, 4014-20.
(6) Parker, D.; Soong, G.; Planet, P.; Brower, J.; Ratner, A J.; Prince, A. Infect Immun 2009, 77, 3722-30.

(7) Pettigrew, M. M.; Fennie, K. P.; York, M. P.; Daniels, J.; Ghaffar, F. Infect Immun 2006, 74, 3360-5.

(8) Uchiyama, S.; Carlin, A. F.; Khosravi, A.; Weiman, S.; Banerjee, A.; Quach, D.; Hightower, G.; Mitchell, T. J.; Doran, K. S.; Nizet, V. J Exp Med 2009, 206, 1845-52.

(9) Xu, G.; Potter, J. A.; Russell, R. J.; Oggioni, M. R.; Andrew, P. W.; Taylor, G. L. J Mol Biol 2008, 384, 436-49.

(10) Gut, H.; King, S. J.; Walsh, M. A. FEBS Lett 2008, 582, 3348-52.

(11) Burmeister, W. P.; Henrissat, B.; Bosso, C.; Cusack, S.; Ruigrok, R. W. Structure 1993, 1, 19-26.

(12) Moustafa, I.; Connaris, H.; Taylor, M.; Zaitsev, V.; Wilson, J. C.; Kiefel, M. J.; von Itzstein, M.; Taylor, G. J Biol Chem 2004, 279, 40819-26.

(13) Newstead, S. L.; Potter, J. A.; Wilson, J. C.; Xu, G.; Chien, C. H.; Watts, A. G.; Withers, S. G.; Taylor, G. L. J Biol Chem 2008, 283, 9080-8.

(14) Xu, G.; Li, X.; Andrew, P. W.; Taylor, G. L. Acta Crystallogr Sect F Struct Biol Cryst Commun 2008, 64, 772-5. 
